\title{
Consumers' Willingness to Try a Robotic Shopping Assistant: The Role of Imagery: An Abstract
}

\author{
Nobuyuki Fukawa and Yu-Shan Huang (Sandy)
}

\begin{abstract}
To win online shoppers back, some brick-and-mortar retailers have started to adopt robotic shopping assistants. Latest robotic shopping assistants can interact with consumers more human-like than before and may enable brick-andmortar retailers to enhance the store experience of consumers and improve profitability. Some researchers argue that this robotic technology may potentially alter the landscape of retailing business in the next decade (Parasuraman and Colby 2015; Bertacchini et al. 2017; Keeling et al. 2013). However, some consumers still show negative responses to robotic shopping assistants. Thus, to take advantage of this technology, more research is needed to understand how consumers respond to this technology (Huang and Rust 2017). In this study, we explore how consumers form imagery and attitude toward a robotic shopping assistant (Study 1). Second, we investigate how the style of a robotic shopping assistant (humanoid vs. nonhumanoid) and its feature types (hedonic vs. utilitarian) affect the imagery, attitude, and willingness to try (Study 2). Our results show that the willingness to try a robotic shopping assistant can be improved through enhancing imagery. Furthermore, our results suggest that the feature types affect imagery and willingness to try only for a humanoid robotic shopping assistant, but not for a nonhumanoid robotic shopping assistant.
\end{abstract}

\footnotetext{
N. Fukawa $(\bowtie)$

Missouri University of Science and Technology, Rolla, MO, USA

e-mail: fukawan@mst.edu

Y.-S. Huang (Sandy)

Northern Michigan University, Marquette, MI, USA

e-mail: yhuang@nmu.edu 